\title{
"El Agroturismo y como se relaciona con el desarrollo turístico en el Distrito de Huayllabamba, Provincia de Urubamba, en el Departamento de Cusco"
}

\author{
"Agroturism and as related to tourism development in the \\ Huayllabamba District, Province of Urubamba, in the Cusco \\ Department"
}

\author{
Dr. Vasquez Ramirez Elvert Vidal / Shirly Ingrid Mota Díaz \\ Facultad de Ciencias Empresariales / Universidad Alas Peruanas \\ Correo-e: e_vasquez@uap.edu.pe
}

\section{RESUMEN}

Objetivo: La Presente investigación tiene como objetivo establecer la relación del agroturismo con el desarrollo turístico en el distrito de Huayllabamba provincia de Urubamba, departamento de Cusco. Materiales y Métodos: La investigación a aplicar, será de acuerdo a una investigación cualitativa con un Diseño descriptivo simple (no experimental), debido a que observaremos como se está llevando a cabo este tipo de turismo y de qué manera se considera un desarrollo turístico beneficioso para la región receptora. El tipo de investigación es, Sustantiva Descriptiva, el corte del tipo de investigación es: transversal, debido a que solo se dará en un momento dado. Se trabajó con una muestra de 67 pobladores de la cual el tipo de método fue probabilístico, debido a que todos tuvieron la posibilidad de ser seleccionados, para desarrollar la observación acerca de la relación del agroturismo con el desarrollo turístico. La técnica a emplearse en la presenta investigación será la observación por medio de la lista de cotejo. Resultado: De los 67 pobladores que desarrollaron la lista de cotejo, se puede observar, que un $43.3 \%$, indico que existe un beneficio económico hacia la comunidad, ya que en temporadas de cosecha y sembrío son las temporadas de mayor afluencia de visitantes y un 59,7\% a nivel alto, han aumentado las oportunidades de progreso para la comunidad, tanto a nivel familiar como regional. Conclusiones: Se concluye que el agroturismo tiene relación con el desarrollo turístico, en el distrito de Huayllabamba, con un $43.3 \%$ de pobladores que índico que existe un beneficio económico hacia la comunidad. Por otro lado, la comunidad cuenta con un personal capacitado de 52,2\%, quienes han sido capacitados en temas de servicio al turista, guiado, rescate primeros auxilios, entre otros conocimientos y con un alto índice de $53.2 \%$ el atractivo se encuentra en condiciones de ser visitada, ya que cuenta con el área y experiencia previa para poder realizarlo. Según: Cedeño (2006). "Es un tipo de turismo rural que se caracteriza por la visita emprendimientos rurales para conocer las actividades agropecuarias, involucrándose en las mismas, lo que puede dar origen a actividades asociadas como: hacienda-hotel, posada, restaurante típico, ventas directas del productor, artesanías y otras actividades de recreación ligadas a la vida cotidiana de los pobladores del campo" (p.29).

Palabras Claves: Agroturismo, Desarrollo turístico. 


\begin{abstract}
Objective: The present research aims to establish the relationship of agrotourism with tourism development in the district of Huayllabamba province of Urubamba, department of Cusco. Materials and Methods: The research to be applied will be according to a qualitative research with a simple descriptive design (non-experimental), because we will observe how this type of tourism is being carried out and how it is considered a beneficial tourism development For the recipient region. The type of research is, Descriptive Noun, the cut of the type of investigation is: transversal, because it will only occur at a given time. We worked with a sample of 67 inhabitants of which the type of method was probabilistic, because all had the possibility to be selected, to develop the observation about the relationship of agrotourism with tourism development. The technique to be used in the present investigation will be the observation by means of the checklist. Results: Of the 67 people who developed the checklist, it can be observed that $43.3 \%$ indicated that there is an economic benefit to the community, since during seasons of harvest and sowing are the seasons of greatest influx of visitors and a 59.7\% at a high level, there has been an increase in the opportunities for community development at both the family and regional levels. Conclusions: It is concluded that agrotourism is related to tourism development, in the district of Huayllabamba, with $43.3 \%$ of inhabitants indicating that there is an economic benefit to the community. On the other hand, the community has a trained staff of $52.2 \%$, who have been trained in matters of tourist service, guided, first aid rescue, among other knowledge, by the municipality of Cusco and with a high index of $53.2 \%$ the attraction is in conditions to be visited, since it has the area and previous experience to be able to do it. According to: Cedeño (2006). "It is a type of rural tourism that is characterized by the visit to rural enterprises to know agricultural activities, getting involved in them, which can give rise to associated activities such as hacienda-hotel, inn, typical restaurant, direct sales of the producer, Handicrafts and other recreational activities linked to the daily life of the rural people "(p.29).
\end{abstract}

Key words: Agrotourism, Tourism development.

\title{
Introducción
}

La descripción del problema es conocer como el agroturismo tiene relación con el desarrollo turístico en el distrito de Huayllabamba, en el departamento de Cusco, en cómo está siendo beneficiada la región, como se está desarrollando el agroturismo en el medio ambiente rural y si cuenta con servicios idóneos que pruebe que este tipo de turismo pueda posicionarse dentro de cusco. El desear conocer como es el desarrollo turístico, si cuenta con lo necesario que pruebe que este tipo de turismo pueda posicionarse dentro del Cusco, mostrara que relación cuenta con el Agroturismo

La presente investigación se elaboró con la finalidad de no solo en la búsqueda de novedades acerca de que tan informados estamos acerca de este nuevo tipo de turismo que se está comenzando en Cusco, sino además conocer qué consecuencias trae este segmento, que puede abarcar mayor empleo, mayores ingresos, pero sobre todo mayor reconocimiento de lo nuestro.

Cedeño (2006). "Es un tipo de turismo rural que se caracteriza por la visita emprendimientos rurales para conocer las actividades agropecuarias, involucrándose en las mismas, lo que puede dar origen a actividades asociadas como: hacienda-hotel, posada, restaurante típico, ventas directas del productor, artesanías y otras actividades de recreación ligadas a la vida cotidiana de los pobladores del campo" (p.29).

El agroturismo en Huayllabamba brinda cinco circuitos: ruta del arado, ruta del maíz, ruta de las haciendas, ruta del trekking, y el circuito vial, dándose una variedad de servicios, en cuanto a la situación en Ecuador como parte de su desarrollo del Plan Estratégico de Desarrollo de Turismo Sostenible para el año 2020, (PLANDETUR 2020) en su diagnóstico se realizó una consultoría de productos, que fue analizada y validada por un grupo de especialistas llamado Grupo de trabajo de mercadeo. Este estudio identificó 11 líneas de producto y sus variedades específicas para el Ecuador. El producto Agroturismo fue uno de los productos prioritarios y potenciales, las variedades que incluye son: haciendas, fincas y plantaciones. Dos productos sobresalen de este análisis: "Paseo de los Sabores, Cacao, Banano, Café" y 
"Ruta de las Flores, plantaciones de flores del Ecuador”, en cambio a nuestros circuitos donde destaca la cosecha y sembrío del maíz.

Según: Diario El País, en la columna Alter Consumismo coordinado por Anna Argemí, el 29 de septiembre del 2015 "En otras ocasiones las comunidades que ofrecen este tipo de turismo se han organizado en redes, disponen de touroperadoras profesionales, lo que les permite brindar un servicio de calidad a los turistas, además de acompañar y fortalecer a las iniciativas comunitarias. Es el caso, por ejemplo, de ACTUAR en Costa Rica, TUSOCO en Bolivia, VIVA ATITLÁN en Guatemala o RUNA TUPARI en Ecuador. En otros casos estas redes $\mathrm{u}$ organizaciones comunitarias han creado alianzas estables con tour-operadoras privadas con las que han establecido una serie de acuerdos y compromisos para garantizar el beneficio y control de las comunidades sobre la actividad turística, como por ejemplo ORIGINS en Argentina que trabaja con la Red de Turismo Campesino de Salta, Toururales, que colabora con la Mesa Nacional de Turismo Rural Comunitario de El Salvador, o Estaçao Gabiraba que está aliada con la Red Tucum en el nordeste de Brasil.

También hay asociaciones o fundaciones que han creado tour-operadoras para ayudar a las comunidades a comercializar su oferta, además de mejorar sus infraestructuras o entrenamiento ante una nueva actividad. Iniciativas como Travolution en Chile destacan en este sentido".

Las variables elegidas fueron: Agroturismo, donde según la Secretaria de Turismo de México (2005) El agroturismo es "La actividad turística en áreas agropecuarias con el aprovechamiento de un medio ambiente rural, ocupada por una sociedad campesina, que muestra y comparte no solo su idiosincrasia y técnicas agrícolas, sino también su entorno natural, cultural y socio-productivo" y Desarrollo Turístico que puede definirse específicamente como la provisión y el mejoramiento de las instalaciones y servicios idóneos para satisfacer las necesidades del turista, definido de una manera más general, puede también incluir efectos asociados, tales como la creación de empleos o la generación de ingresos" (Pearce 1991: 14)

Siendo el objetivo general de la investigación establecer la relación del agroturismo con el desarrollo turístico en el distrito de Huayllabamba provincia de Urubamba, departamento de Cusco, ya que este tipo de turismo se está haciendo cada vez más conocido dentro del mercado.

\section{Materiales y métodos}

Se trabajó con una muestra de 67 pobladores de la cual el tipo de método fue probabilístico, debido a que todos tuvieron la posibilidad de ser seleccionados, para desarrollar la observación acerca de la relación del agroturismo con el desarrollo turístico.

El diseño utilizado para la investigación es diseño Descriptivo - simple (no experimental), debido a que observaremos como se está llevando a cabo este tipo de turismo y de qué manera se considera un desarrollo turístico beneficioso para la región receptora.

Según Palella et Martins (2004), expresan que el diseño de la investigación "se refiere a la estrategia que adopta el investigador para responder al problema, dificultad o inconveniente planteado en el estudio".

El tipo de investigación es Sustantiva Descriptiva, el corte del tipo de investigación: es transversal, porque solo se dará en un momento dado y el nivel de Investigación es Descriptiva, porque de acuerdo al problema planteado, referido a la relación del agroturismo con el desarrollo turístico en el distrito de Huayllabamba, será necesario realizar una investigación descriptiva para poder dar diversas soluciones acerca de los beneficios que contrae la relación del agroturismo con el desarrollo turístico.

Según ICT-Alianza TRC (2005) "Experiencias turísticas planificadas e integradas sosteniblemente al medio rural y desarrolladas por los pobladores locales organizados para beneficio de la comunidad".

La técnica utilizada para esta investigación es la observación, y el instrumento a emplear es la hoja de cotejo para evaluar el punto de vista de los pobladores de como que el agroturismo toma ventaja en cuanto a un desarrollo turístico en su región. Los datos fueron recopilados y analizados con el sistema estadístico o software el SPSS versión 22.

\section{Resultados}

De los 67 turistas que desarrollaron la observación, se pudo observar que De los 67 pobladores que desarrollaron la lista de cotejo, se puede observar, que un $43.3 \%$, indico que existe un beneficio económico hacia la comunidad, ya que en temporadas de cosecha y sembrío son las 
temporadas de mayor afluencia de visitantes y un $59,7 \%$ a nivel alto, han aumentado las oportunidades de progreso para la comunidad, tanto a nivel familiar como regional. (Fig. 1)

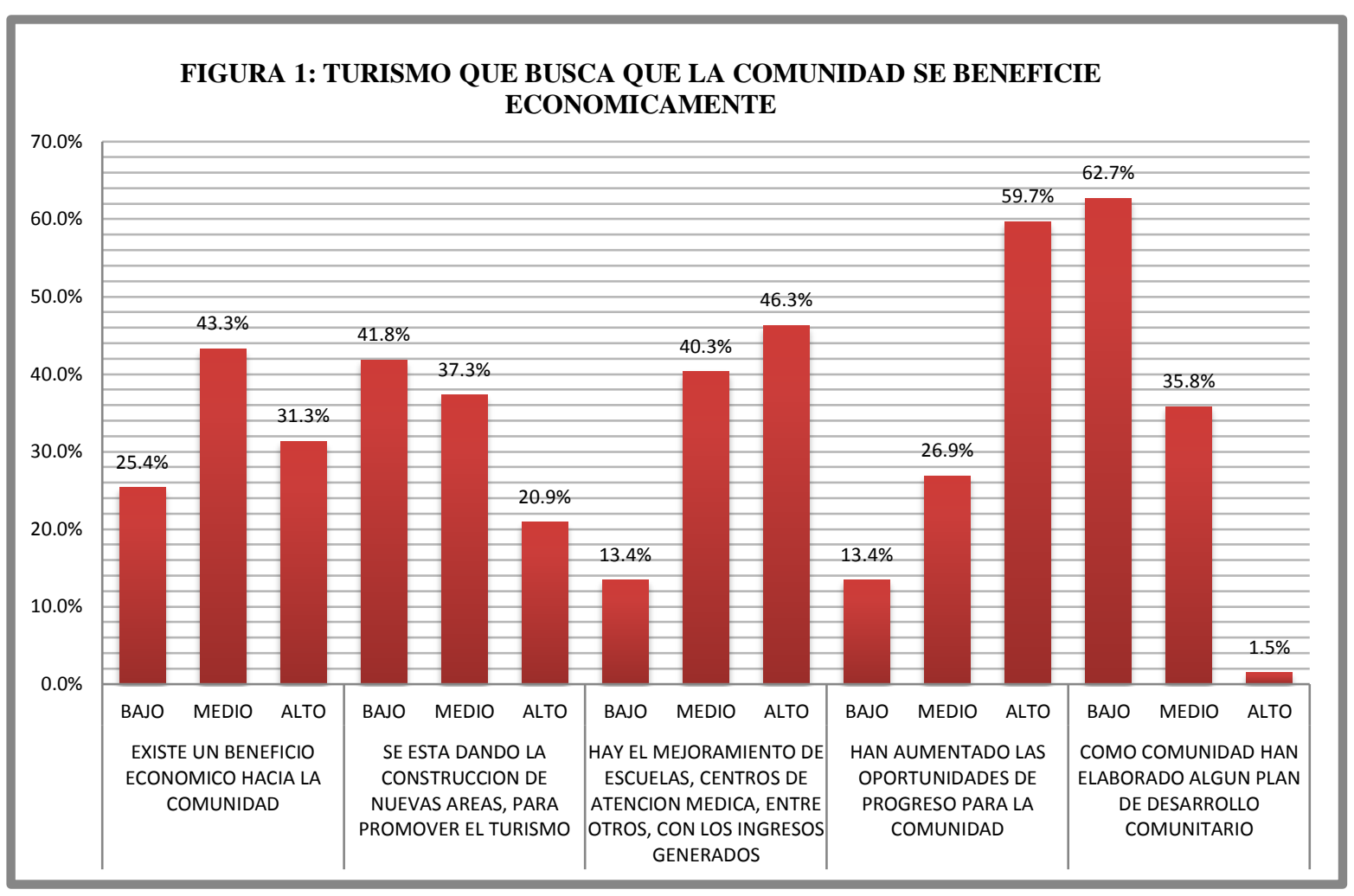

Fuente: Elaboración Propia

\section{Discusión}

El fruto de la investigación de campo, indican resultados satisfactorios, donde se concluyó que el agroturismo tiene relación con el desarrollo turístico, en el distrito de Huayllabamba, con un $43.3 \%$ que indica que existe un beneficio

económico hacia la comunidad, ya que en temporadas de cosecha y sembrío son las que más afluencia atrae y un 59,7\% a nivel alto, han aumentado las oportunidades de progreso para la comunidad, tanto a nivel familiar como regional. Por otro lado, la comunidad cuenta con un personal capacitado de $52,2 \%$, quienes han sido capacitados en temas de servicio al turista, guiado, rescate primeros auxilios, entre otros conocimientos y con un alto índice de $53.2 \%$ el atractivo se encuentra en condiciones de ser visitada, ya que cuenta con el área y experiencia previa para poder realizarlo.

Estas conclusiones son similares a los de Olivera, Maylin; Negrón, cesar; Muñoz, José y Paucar, Emilio. "Planeamiento Estratégico para el Turismo Rural Comunitario en Cuatro Lagunas
Cusco, Pontificia Universidad Católica del Perú - Escuela de Negocios, 2008”, cuyas conclusiones fueron: El turismo rural comunitario en el Perú está representado por las experiencias de las regiones de Puno, Cusco y Madre de Dios, de las mismas que han sido puestas en marcha con el apoyo de instituciones públicas y privadas así como la participación de la población y sus actores económicos, sin embargo, esta oferta está basada principalmente en el aprovechamiento de ventajas comparativas, como: el legado cultural, recursos naturales y arqueológicos y no en la creación de ventajas competitivas, como el desarrollo de capacidades de los recursos humanos, hospedajes, transportes y hospedajes, así como una cultura empresarial con visión de futuro. Tal y como lo indica los autores en las conclusiones dadas, las experiencias de un turismo rural están representadas en las regiones de Puno, Madre de Dios y cusco, ya que cuentan con el espacio y el apoyo con entidades públicas y el entusiasmo de los pobladores por brindar este tipo de experiencias en su entorno natural.

Pavón Álvarez, A. "Proyecto de Agroturismo como una alternativa de Turismo Rural en la 
Hacienda Italia Esperanza, Cantón Valencia, Provincia de los Ríos. Universidad de Guayaquil - Facultad de Comunicación Social, 2015”, dice que la información recopilada permitió conocer algunos datos sobre el turismo rural, agroturismo, producto turístico e información de la localidad donde se encuentra la hacienda. En conjunto con la elaboración de encuestas y entrevistas con los visitantes y la empresa privada de la localidad que permitió realizar una propuesta acorde a los gustos y preferencias de los turistas. Lo que permite concluir que es un proyecto viable que produce expectativa a los turistas y crea un proyecto amigable con el medio ambiente. Promueve la inversión de la empresa privada y para que la comunidad local sea consciente de las riquezas naturales con la que cuentan. Indicando claramente que el agroturismo es una forma de que la comunidad receptora se beneficie $y$ aproveche sus recursos naturales, tal como sucede en el distrito de Huayllabamba.

Según Segura Viteri, J., "Propuesta para la Implantación de Agroturismo en la Finca Doris Rivera en el Cantón Yaguachi, provincia del Guayas, para el Desarrollo turístico y económico del sector, Universidad Tecnológica Equinoccial - Facultad de Turismo y Preservación Ambiental, Hotelería y Gastronomía, 2010, "Según las entrevistas al turista nacional, el agroturismo es muy acogido por los turistas de Quito especialmente por las plantaciones de café y frutas en general. La degustación de productos de cultivo y cosecha de productos son las actividades que más los atraen. El tiempo de estadía dispuesto por los turistas para permanecer en una finca es mayor a un día. En cuanto a reservaciones, los quiteños prefieren realizar las reservaciones directamente con el personal de la finca y no mediante agencias de viajes.

En cuanto a los turistas extranjeros el agroturismo en una finca es una opción muy aceptada, el producto en el que se muestra más interés es en el cacao y las frutas en general. Las actividades preferidas a realizar en una finca agroturistica son la degustación de productos y el trabajo de cultivo y cosecha de la fincan y en cuanto a la estadía realizarían la visita de un día en la finca". Según las conclusiones el agroturismo es un fuerte potencial para cada región donde se piensa aplicar, ya que no necesita de una arquitectura de por medio, solo brindar un buen servicio acompañado del área natural y el resultado de la elaboración con productos naturales.

Según Padilla, A., Ramírez, J., "Plan de Desarrollo Turístico Rural Comunitario Sostenible para el Municipio de San Luis del
Carmen, Departamento de Chalatenango, Universidad de el Salvador - Escuela de Ingeniería Industrial, 2014, las conclusiones formuladas fueron: el turismo no convencional como turismo-rural y turismo-comunitario son una oportunidad para el sector municipio de San Luis del Carmen pueda desarrollarse, la implementación de este tipo de turismo podría contribuir directamente al desarrollo de áreas rurales a través del aprovechamiento y conservación de los recursos con los que estos cuentan, con el fin de que este se convierta en un medio sostenible y permita la participación de todos y todas procurando la equidad de género y contribuyendo al mejoramiento de la calidad de vida de los mimos. Corroborando nuevamente que el agroturismo es un medio donde se da la conservación de los recursos y con este fin se convierta en un medio sostenible para los participantes beneficiados.

Según Borbor Pozo, P.D., "Plan Estratégico para el Desarrollo del Turismo Comunitario en la Comuna Ayangue, Parroquia Colonche del Cantón de Santa Elena, Provincia de Santa Elena, año 2014, Universidad Estatal Península de Santa Elena - Escuela de Hotelería y Turismo, 2014, a través de la investigación se valoró la situación turística, poseyendo una buena infraestructura que sirve de acceso al uso de la oferta. Siendo los atractivos de sitio natural y gastronómico ocupan la jerarquía más alta.

Se planteó el plan de acción, en el que se proyecta el Plan Estratégico para el desarrollo del turismo comunitario en la comuna Ayangue, año 2013-2018, el mismo que persigue consolidar el turismo comunitario como eje articulador del desarrollo de Ayangue en el marco de la sostenibilidad, de forma que contribuya a garantizar a los habitantes el acceso a una vida digna y a generar las condiciones necesarias para mejorar la competitividad en la región.

Además, se consideró a los GDA, s, dependencias del gobierno central, ONGs, microempresas y empresas comunitarios como responsables del turismo comunitario por competencias que les habilitan otorgar ayuda a la comunidad de Ayangue.

El resultado del estudio concuerda con los de Olivera, Maylin; Pavón Álvarez; Segura Viteri y Padilla, A., que concluyen que el agroturismo es una fuente de avance para la población, a través de un turismo con desarrollo turístico sostenible con beneficios que permitan el avance de la población receptora que brinda el servicio. 


\section{Conclusiones}

Se concluye que el agroturismo tiene relación con el desarrollo turístico, en el distrito de Huayllabamba, con un $43.3 \%$ que indica que existe un beneficio económico hacia la comunidad, ya que en temporadas de cosecha y sembrío son las que más afluencia atrae y un $59,7 \%$ a nivel alto, han aumentado las oportunidades de progreso para la comunidad, tanto a nivel familiar como regional. Por otro lado, la comunidad cuenta con un personal capacitado de 52,2\%, quienes han sido capacitados en temas de servicio al turista, guiado, rescate primeros auxilios, entre otros conocimientos y con un alto índice de $53.2 \%$ el atractivo se encuentra en condiciones de ser visitada, ya que cuenta con el área y experiencia previa para poder realizarlo. Según: La OMT (2009). "Las actividades turísticas que se realizan en el espacio rural y que tienen como fin interactuar con la vida rural, conocer las tradiciones y la forma de vivir de la gente y los atractivos de la zona".

\section{Referencias bibliográficas}

[1] Eumed.Net. Tipos de Turismo. Recuperado de: http://www.eumed.net/librosgratis/2012b/1199/tipos_de_turismo.html.

[2] Palella y Martins (2004: Párr.18). Diseño de la Investigación. Recuperado de: http://www.eumed.net/tesisdoctorales/201 2/zll/metodologiainvestigacion.html.

[3] Ministerio de Turismo. PlandeTur 2020. Recuperado de:

http://www.undp.org.ec/odm/planes/pland etur.pdf

[4] El País (2015). Nace el Catalogo Latinoamericano de Turismo Rural Comunitario. Recuperado de: http://elpais.com/elpais

[5] Tnews (2009). Lanzan Proyecto Pionero de Agroturismo en el Perú. Recuperado de: http://www.tnews.com.pe

[6] Pavón Álvarez, Ana Jezabel (2015). Proyecto de Agroturismo como
Alternativa de Turismo Rural en la Hacienda Italia. Recuperado de: http://www.tnews.com.pe

[7] Pavón Álvarez, Ana Jezabel (2015). Proyecto de Agroturismo como Alternativa de Turismo Rural en la Hacienda Italia. Recuperado de: http://repositorio.ug.edu.ec/bitstream/redu g/

[8] Olivera Lazo, Maylin Vanesa; Negrón Ortiz, Cesar Augusto; Muñoz Delgado, José; Paucar Aguirre, Emilio (2008). Planeamiento para el Turismo Rural Comunitario en Cuatro Lagunas Cusco. Recuperado de: http://tesis.pucp.edu.pe/repositorio/handle /123456789/1624.

[9] Padilla, Andrea., Ramírez, Jonathan. y Repreza, Laura (2014). Plan de Desarrollo Turístico Rural Comunitario Sostenible para el Municipio de San Luis del Carmen. Recuperado de: http://ri.ues.edu.sv/5583/1/Plan\%20de\%2 0desarrollo\%20tur\%C3\%ADstico\%20rur al\%20comunitario\%20sostenible\%20para $\% 20$ el\%20municipio\%20de\%20San $\% 20 \mathrm{~L}$ uis $\% 20$ del $\% 20$ Carmen $\% 2 C \% 20$ departam ento\%20de\%20Chalatenango.pdf

[10] Segura Viteri, José Daniel (2010). Propuesta para la implementación de agroturismo en la Finca Doris Rivera. Recuperado de:

http://www.repositorio.ute.edu.ec/bitstrea m/123456789/13336/1/43842_1.pdf

[11] Segura Viteri, José Daniel (2010). Propuesta para la implementación de agroturismo en la Finca Doris Rivera. Recuperado de:

http://www.repositorio.ute.edu.ec/bitstrea $\mathrm{m} / 123456789 / 13336 / 1 / 43842 \_1 . p d f$

[12] Yumisaca Tuquinga, Jhony (2014). Plan Estratégico para el Desarrollo del Turismo Comunitario en la Comuna Ayangue. Recuperado de: http://repositorio.upse.edu.ec/bitstream/46 000/1756/1/BORBOR\%20POZO\%20DA VID.pdf 